\title{
Damping Characteristics of Flexural Vibration for Partially Covered Beams with Constrained Viscoelastic layers*
}

\author{
Hiromi DEWA**, Yohji OKADA** \\ and Bunsyu NAGAI**
}

\begin{abstract}
A beam partially covered with a constrained viscoelastic layer shows superior damping capacity to that of a fully covered beam, depending on the beam geometry and the properties of the viscoelastic layer. This damping effect, the so-called partial cover effect, however, had not been examined in detail until now. In this paper, the strain energy analysis of the three types of partially covered five-layered beam is carried out in order to explain the damping mechanism. The results of analysis for the center-covered beam and the end-constrained beam indicate that the partial cover effect is caused by the shear strain energy dissipation at the outer edges of the viscoelastic layer, and also, such energy dissipation depends on the tensile stiffness of the constraining layer. The separately covered beam has a superior damping capacity to that of the center-covered beam with the same cover ratio, due to the energy dissipation of the inner edges of the viscoelastic layers. These damping characteristics of the three types of beam presented are confirmed by experimental results.
\end{abstract}

Key Words : Vibration, Damping, Partial Cover Effect, Partially Covered Beams, Loss Factor, Strain Energy Analysis, Viscoelastic Layer

\section{Introduction}

Laminated steel sheets composed of elastic and viscoelastic layers, especially the sandwich type steel sheets with viscoelastic core well known as the "damping steel sheet", have been utilized as an excellent damping structural material against acoustic noise snd mechanical vibration. These damping steel sheets are now used up to $\mathbf{4 0}$ percent in the oil pan of small diesel engine trucks ${ }^{(1)}$ in the domestic automobile industries.

Since the earliest studies on the damping characteristics of the two layered beam by Oberst ${ }^{(2)}$ and the sandwich beam by $\mathrm{Kerwin}^{(3)}$, a large number of studies on sandwich beams and plates have been reported $^{(4) \sim(6)}$. In these previous studies, the partial cover effect which a partially covered treatment by the constrained viscoelastic layer on the main beam is superior to the fully covered beam in damping capacity, had been indicated by Nokes and Nelson ${ }^{(7)}$. This

\footnotetext{
* Received 28th March, 1990. Paper No. 88-0900B

** Faculty of Engineering, Ibaraki University, 4-12-1, Nakanarusawa-cho, Hitachi 916, Japan
}

effect was subsequently confirmed by more exact theories and detailed experiments by a few authors for the beam ${ }^{(8) \sim(10)}$, plate ${ }^{(11)}$ and circular plate ${ }^{(12)}$.

The damping mechanism for this effect, however, is not explained clearly so far, even though the presumptions ${ }^{(7)(9)}$ that this effect is attributed to the increase of shear deformations in the outer edges of the viscoelastic lsyer has been given.

In this paper, a strain energy analysis was performed for the center-covered beam and the end-constrained beam, as shown in Fig. 1(a) and Fig. 1(b), in order to explain the partial cover effect based on the above presumptions.

Furthermore, in the symmetric mode of flexural vibration, a neighboring part of anti-node point of the viscoelastic layer almost does not attribute to strain energy dissipation because of a small shear deformation. If we assume that the partial cover effect depends on the above presumptions, the energy dissipation of the separately covered beam, as shown in Fig. 1(c), seems to increase more than that of the center-covered beam due to the energy dissipation at the inner edges. Then, we also examined the case for the separately covered beam. 
These damping characteristics estimated from the above analytical results are verified by some experimental results.

\section{Analysis}

The approximate analysis by Nokes and Nelson ${ }^{(7)}$, in which they assumed that the partially covered beam keeps the normal modes as a homogeneous beam, does not agree well with the experimental results if the bending stiffness of the constraining layer as well as the shear modulus of the viscoelastic layer become greater. However, this snalytical procedure is simple, and it seems that the damping characteristics of partially covered beam can be estimated roughly by this approximate analysis.

Then, we applied the approximate analysis to the three types of partially covered beam, as shown in Fig. 1. The center covered beam in Fig. 1(a) is the usual partially covered beam, and the end-constrained beam in Fig. 1 ( b ) is the beam which constrains shear deformations of the viscoelastic layer ends. The separately covered beam in Fig. 1(c) is the beam which is divided the viscoelastic layer only into two parts at its beam center.

The following assumptions are made through the analysis :

(1) No slippage occurs in the interface between each layer.

(2) Young's modulus of the viscoelastic layer is small in comparison with that of the constraining layer ; that is, the axial force of the viscoelastic layer can be ignored.

(3) The transverse displacement in each layer is

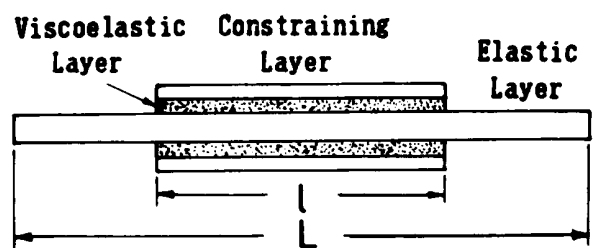

(a) Center-Covered Bean

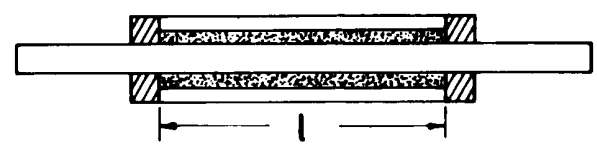

(b) End-Constrained Bea:

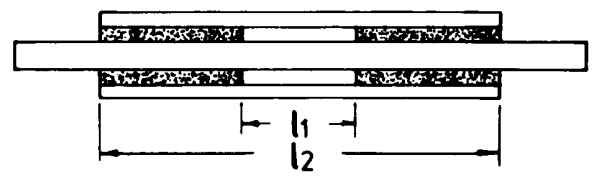

(c) Separately Covered Bean

Fig. 1 Partially covered five-layered beams the same, and the flexural vibration keeps the normal mode.

(4) The damping of the beam depends on only the shear strain energy dissipation in the viscoelastic layer.

(5) The elastic and constraining layers are treated as Bernoulli-Euler beam, then the inertia of rotation of the beam is ignored.

\subsection{Basic equation}

The damping characteristics of the laminated beam with viscoelastic layers depend on factors such as the geometrical beam composition, the material constants, the boundary conditions, and the mode numbers. In order to explain a complicated damping mechanism of the partial cover effect, simpler beam conditions should be provided. We consider thus the case of a simply supported beam and its fundamental mode.

In Fig. 2, the axial force of the elastic layer becomes $F_{3}=0$ due to the geometrical symmetry of the beam. If we consider assumption ( 2 ), the equation of equilibrium for the axial forces per unit beam width in the $x$-direction is given as

$$
2 E_{c} t_{c}\left(\frac{d u_{1}}{d x}+\frac{d u_{5}}{d x}\right)=0
$$

Taking the origin of the coordinates at the geometrical center of the beam, the following equation with respect to the axial displacements of the constraining layers, $u_{1}$ and $u_{5}$, is derived from Eq. (1) and the geometrical relationship in Fig. 2 :

$$
-u_{1}=u_{5}=\left\{t_{3} \phi-2 t_{v}(\phi-\phi)+t_{c} \phi\right\} .
$$

Then, the shear strain $\phi$ in the viscoelastic layer is as follows,

$$
\psi=\frac{1}{4 t_{v}}\left(u_{1}-u_{5}+2 h \phi\right)=-\frac{1}{2 t_{v}}\left(u_{5}-h \phi\right),
$$

where $h=t_{3}+2 t_{v}+t_{c}$

Considering Eq. ( 2 ) and the equilibrium of the axial force of the upper constraining layer and the shear force of the viscoelastic layer; $d F_{5} / d x=-G_{v} \psi$, the following equation is given :

$$
\frac{d^{2} u_{5}}{d x^{2}}=\frac{G_{v}}{4 t_{v} t_{c} E_{c}}\left(u_{5}-h \phi\right) \text {. }
$$

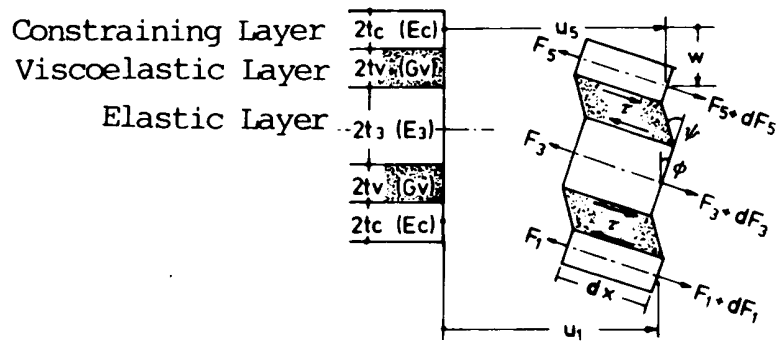

Fig. 2 Deformation of the five-layered beam 
Rewriting Eq. ( 3 ) by using the dimensionless constant $R=G_{v} L^{2} /\left(4 t_{v} t_{c} E_{c}\right)$ and the $x$ co-ordinate $\xi=x / L$, the following basic equation for $u_{5}$ is obtained :

$$
\frac{d^{2} u_{5}}{d \xi^{2}}-R u_{5}=-R h \phi .
$$

From assumption ( 3 ), the following boundary conditions at the fundamental mode are applied to the present beam :

$$
\begin{aligned}
& w=0, \quad d^{2} w / d \xi^{2}=0(\xi= \pm 1 / 2) \\
& d w / d \xi=0, d^{3} w / d \xi^{3}=0 \quad(\xi=0) .
\end{aligned}
$$

Then, the transverse displacement $w$ and the slope $\phi$ are easily given as

$$
w=w_{0} \cos \lambda \xi, \phi=-\frac{\lambda w_{0}}{L} \sin \lambda \xi .
$$

A general solution of Eq. (4) is obtained by substituting $\phi$ into Eq. ( 4$)$ as the following :

$$
u_{5}=A_{1} \sinh \sqrt{R} \xi+A_{2} \cosh \sqrt{R} \xi-a \sin \lambda \xi,
$$

where $a=R h \lambda w_{0} / L\left(\lambda^{2}+R\right), \lambda=\pi$, and $w_{0}$ is the transverse displacement at the steady state.

In the following, the strain energy components for three types of partially covered beam are determined based on the axial displacement $u_{5}$ in Eq. ( 6 ).

\subsection{Center-covered beam}

The boundary conditions for the constraining layer in Fig. 1 (a) are given as follows (denoting the cover ratio ; $\theta=l(L)$,

$$
u_{5}=0(\xi=0), d u_{5} / d \xi=0(\xi= \pm \theta / 2),
$$

since there is no axial displacement at the anti-node and no normal stress at the layer ends, respectively. Considering the above conditions, $u_{5}$ and $\psi$ are determined from Eq. ( 6$)$ and Eq. (2) as follows :

$$
\begin{aligned}
& u_{5}=a\left(\frac{\lambda q}{\sqrt{R}} \sinh \sqrt{R} \xi-\sin \lambda \xi\right), \\
& \psi=\frac{\lambda a}{2 t_{v}}\left(\frac{q}{\sqrt{R}} \sinh \sqrt{R} \xi+\frac{\lambda}{R} \sin \lambda \xi\right),
\end{aligned}
$$

where

$$
q=\frac{\cos (\lambda \theta / 2)}{\cosh (\sqrt{R} \theta / 2)}
$$

The strain energy components of the center-covered beam, such as the shear strain energy $\bar{U}_{s}$ in the viscoelastic layer, the tensile strain energy $\bar{U}_{e}$ and that of $\bar{U}_{f}$ due to bending in the constraining layer, and that of $\bar{U}_{F}$ due to bending in the elastic layer, are determined from the following equations, using Eqs. ( 7 ), ( 8 ), and Eqs. ( 5 ):

$$
\left.\begin{array}{l}
\bar{U}_{s}=4 t_{v} G_{v} L \int_{0}^{\theta / 2} \phi^{2} d \xi \\
\bar{U}_{e}=\frac{4 E_{c} t_{c}}{L} \int_{0}^{\theta / 2}\left(\frac{d u_{5}}{d \xi}\right)^{2} d \xi \\
\bar{U}_{f}=\frac{2 E_{c} I_{c}}{L^{3}} \int_{0}^{\theta / 2}\left(\frac{d^{2} w}{d \xi^{2}}\right)^{2} d \xi \\
\bar{U}_{F}=\frac{E_{3} I_{3}}{L^{3}} \int_{0}^{1 / 2}\left(\frac{d^{2} w}{d \xi^{2}}\right)^{2} d \xi
\end{array}\right\},
$$

( $I_{C}$ and $I_{3}$ are the second moments of inertia of the constraining and the elastic layer, respectively).

The composite loss factor $\eta_{c}$ of the partially covered beam is determined from the ratio of the imaginary to the real part of the total strain energy stored in the entire beam after replacing the shear modulus $G_{v}$ with the complex shear modulus $G_{v}^{*}=$ $G_{v}(1+i \eta)$, where $\eta$ is the loss factor of the viscoelastic material. However, such calculations are more complicated, so we applied the approximate method in which the maximum strain energy dissipated in the entire beam pera cycle is equivalent to those in the viscoelastic layers. Then, the composite loss factor is determined from the following equation :

$$
\eta_{c}\left(\bar{U}_{s}+\bar{U}_{e}+\bar{U}_{f}+\bar{U}_{F}\right)_{\max }=\eta \bar{U}_{s \max } .
$$

\subsection{End-constrained beam}

The end-constrained beam mounted with small massless chips at its viscoelastic layer ends is assumed to constrain shear deformation only of the viscoelastic layers. In this case, the boundary conditions are given as

$$
u_{5}=0(\xi=0), \phi=0(\xi= \pm \theta / 2) .
$$

Considering these conditions in Eq. ( 6 ), and using Eq. (2) and Eq. (5), the axial displacement $u_{5}$ and the shear strain $\psi$ are given as

$$
\begin{aligned}
& u_{5}=-a\left(\frac{\lambda^{2} p}{R} \sinh \sqrt{R} \xi+\sin \lambda \xi\right), \\
& \psi=\frac{\lambda^{2} a}{2 t_{v} R}(p \sinh \sqrt{R} \xi+\sin \lambda \xi),
\end{aligned}
$$

where

$$
p=\frac{\sin (\lambda \theta / 2)}{\sinh (\sqrt{\bar{R}} \theta / 2)} .
$$

Both strain energies $\bar{U}_{F}$ and $\bar{U}_{f}$ in this beam are equivalent to those of the center-covered beam from the assumption ( 3 ), and $\bar{U}_{e}$ and $\bar{U}_{s}$ as well as $\eta_{c}$ are determined from Eqs. ( 9 ) and (10), respectively.

\subsection{Separately covered beam}

In the separately covered beam in Fig. 1(c), the girder part of the constraining layer is subjected to a bending moment due to the shear force arising at the interface. If we ignore such bending moment under an assumption of a small amplitude of vibration, the sxial force in the girder is kept constant. Then, $u_{5}$ can be represented as

$$
\bar{u}_{5}=A_{3} \xi+A_{4} \text {. }
$$

Denoting the locations of both covered ends in Fig. 1 (c) as $\theta_{1}=l_{1} / L$ and $\theta_{2}=l_{2} / L$, the boundary conditions are as follows :

$$
\bar{u}_{5}=0(\xi=0), d u_{5} / d \xi=0\left(\xi= \pm \theta_{2} / 2\right) .
$$

The coefficients $A_{1}, A_{2}$ and $A_{4}$ in Eq. (6) and Eq. (13) are determined from above conditions, and also $A_{3}$ in Eq. (13) is given by the following condition of continuity in the constraining layer :

$$
\bar{u}_{5}=u_{5}\left(\xi= \pm \theta_{1} / 2\right) \text {. }
$$


Then, the displacement in the constraining layer is given by $u_{5}$ and $\bar{u}_{5}$ as the following :

$$
\begin{aligned}
& u_{5}=a\left(\frac{\lambda q_{2}}{\sqrt{R}} \sinh \sqrt{R} \xi-\sin \lambda \xi\right), \\
& \bar{u}_{5} \frac{2 a}{\theta_{1}}\left\{\frac{\lambda q_{2}}{\sqrt{R}} \sinh \left(\sqrt{R} \theta_{1} / 2\right)-\sin \left(\lambda \theta_{1} / 2\right)\right\} \xi,
\end{aligned}
$$

where

$$
q_{2}=\frac{\cos \left(\lambda \theta_{2} / 2\right)}{\cosh \left(\sqrt{R} \theta_{2} / 2\right)} .
$$

Substituting $u_{5}$ of Eq. (14) into Eq. (2), the shear strain $\phi$ is given as

$$
\phi=\frac{\lambda a}{2 t_{v}}\left(\frac{q_{2}}{\sqrt{R}} \sinh \sqrt{R} \xi+\frac{\lambda}{R} \sin \lambda \xi\right) .
$$

Then, the strain energy components $\bar{U}_{s}$ and $\bar{U}_{e}$ are given as the following :

$$
\left.\begin{array}{rl}
\bar{U}_{s} & =4 t_{v} G_{v} L \int_{\theta_{1} / 2}^{\theta_{2} / 2} \psi^{2} d \xi \\
\bar{U}_{e} & =4 t_{c} E_{c} L\left\{\int_{0}^{\theta_{1} / 2}\left(\frac{d \bar{u}_{5}}{d \xi}\right)^{2} d \xi\right\} \\
& \left.+\int_{\theta_{1} / 2}^{\theta_{2} / 2}\left(\frac{d u_{5}}{d \xi}\right)^{2} d \xi\right\}
\end{array}\right\} .
$$

The other strain energy components $\bar{U}_{F}$ and $\bar{U}_{f}$ are equivalent to those of the center-covered beam in Eq. ( 9 ), and $\eta$ is given also by Eq. (10).

In this beam, if the girder part has a longer span, a coupled transverse vibration arises between the part and the elastic layer. But we ignore such vibration under a consideration of the fact that both layers are transversely coupled with each other, as mentioned in the later experimental procedure.

\section{Calculation Results of the Shear Strain Energy and the Composite Loss Factor}

\section{1 Center-covered beam}

In the following calculations, the elastic and the constraining layers are of the same material $\left(E_{3}=E_{c}\right.$

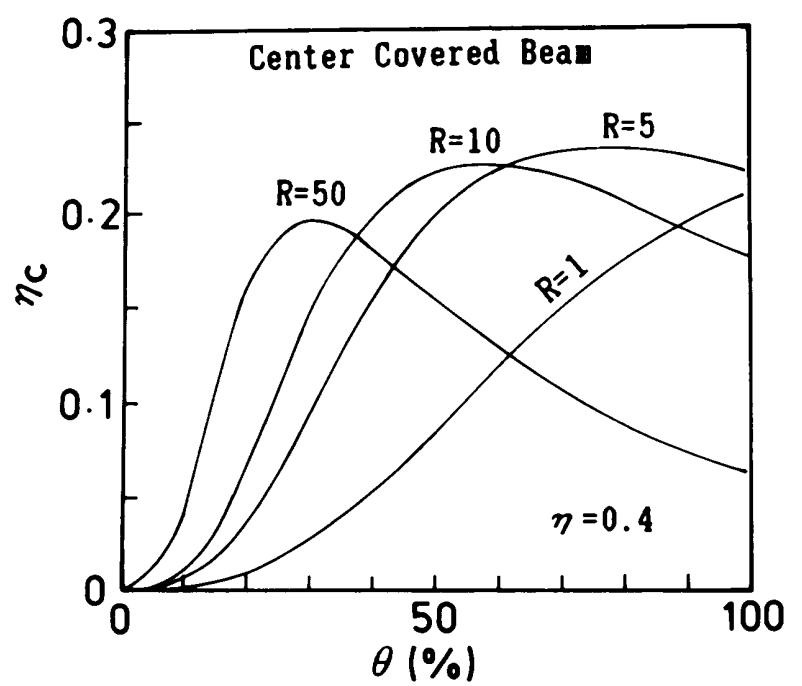

Fig. 3 Composite loss factor of the center-covered beam
$=206 \mathrm{GPa}$ ), and the other numerical values are given as : $t_{3}=1 \mathrm{~mm}, t_{c}=t_{v}=0.5 \mathrm{~mm}, L=900 \mathrm{~mm}$, and $\eta=0.4$. Then, the value of parameter $R$ depends on only the shear modulus $G_{v}$ of the viscoelastic layer.

The calculated results of $\eta_{c}$ for the center-covered beam are shown in Fig. 3 as a function of the cover ratio $\theta$. The low value of $R(R=1)$ does not show a peak value of $\eta_{c}$; however, the higher value of $R(R>5)$ maximizes $\eta_{c}$ at the cover ratio lower than the full cover ratio.

In order to explain the difference of the above damping characteristics, the calculated results for the total strain energy components $\left(\bar{U}_{s}, \bar{U}_{e}, \bar{U}_{F}\right.$, and $\left.\bar{U}_{f}\right)$ and those densities $\left(U_{s}, U_{e}, U_{F}\right.$, and $\left.U_{f}\right)$ per unit beam length of the fully covered and the $60 \%$-covered

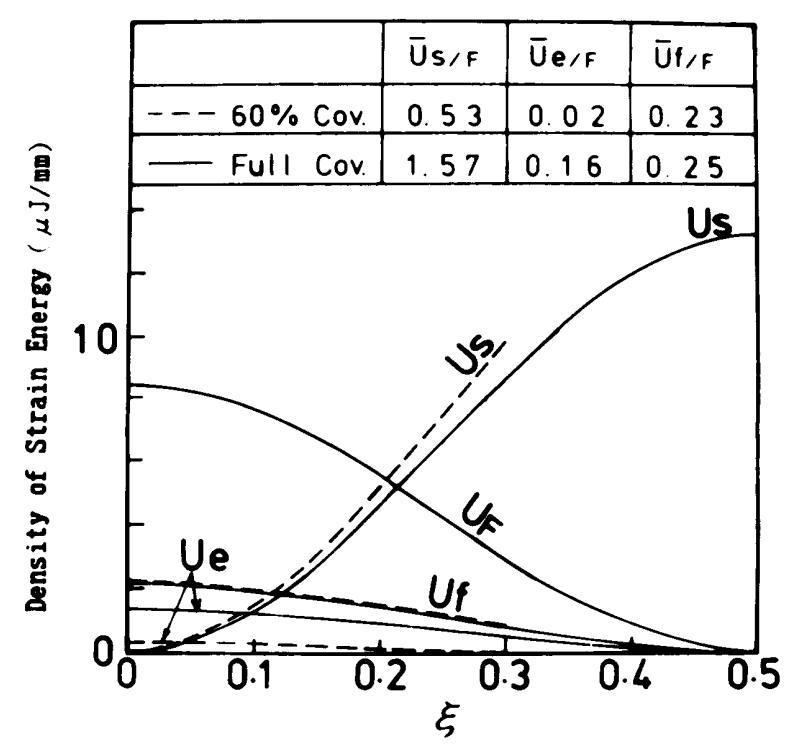

(a) In the case of $R=1$

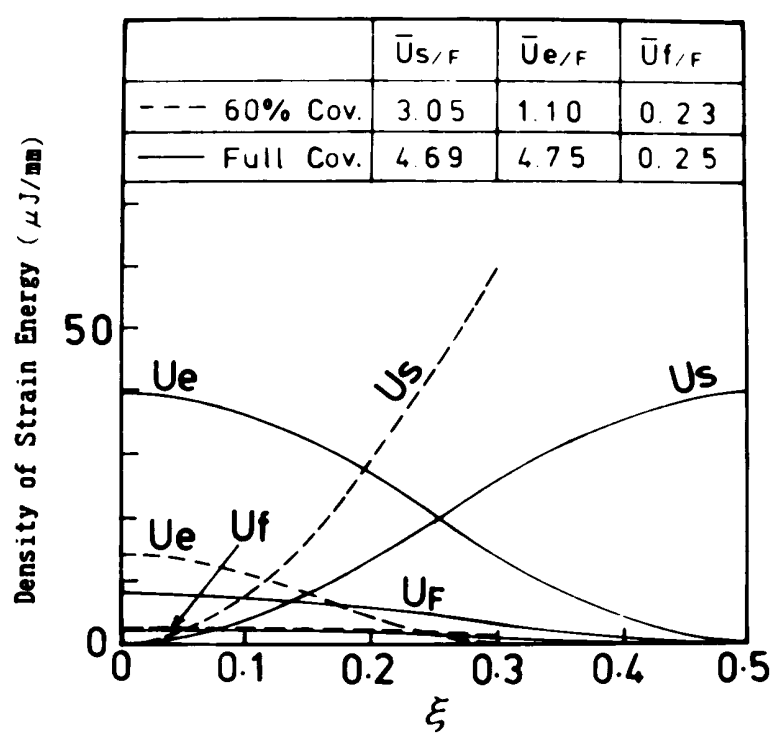

(b) In the case of $R=10$

Fig. 4 Strain energy and its densities of the center-covered beam 
beams are shown in Fig. 4(a) and Fig. 4(b) for the typical cases of $R=1$ and $R=10$. The values of total energy vatio of the table in Fig. 4 give the normalized values divided by $\bar{U}_{F}$ as shown by those suffixes. The former case $(R=1)$ shows no peak value of $\eta_{c}$, and the later case $(R=10)$ maximizes $\eta_{c}$ at the $60 \%$-cover ratio as shown in Fig. 3.

In Fig. 4( b), the value of $U_{s}$ at the covered end $(\xi=0.3)$ of the $60 \%$-covered beam increases more than two times that at the same location of the fully covered beam. Furthermore, the value of $U_{e}$ of the $60 \%$ covered beam decreases more than that of the fully covered beam over the entire covered range. These results are attributed to decrease of the axial displacement of the constraining layer due to the cutting off of the viscoelastic layer ends of the fully covered beam, and this decrease causes, to a great extent, the increase of the shear strain in the remaining viscoelastic layer ends. Here, the values of $U_{F}$ and $U_{f}$ are relatively smaller than those of $U_{s}$ and $U_{e}$, as shown in Fig. 4( b). Therefore, the damping characteristics mainly depend on strain energies of both $\bar{U}_{s}$ and $\bar{U}_{e}$.

On the other hand, in the case of $R=1$, as shown in Fig. $4(\mathrm{a})$, the values of $U_{s}$ at $\xi=0.3$ of both the $60 \%$-covered and the fully covered beam are very close. Such a small difference is caused by the fact that the axial displacement $u_{5}$ becomes sufficiently small due to the low shear stress in the viscoelastic layer ; then, cutting off the covered ends result in little effect on the axial displacement of $\boldsymbol{u}_{5}$.

From the comparison of the damping characteristics between the cases for both $R=1$ and $R=10$, the partial cover effect can be explained to be caused by the shear strain energy dissipation in the neighbor the viscoelastic layer ends. This energy dissipation also depends on both the shear and the tensile stiffnesses of the viscoelastic and the constraining layers.

3. 2 End-constrained beam

In order to confirm the previous explanation of

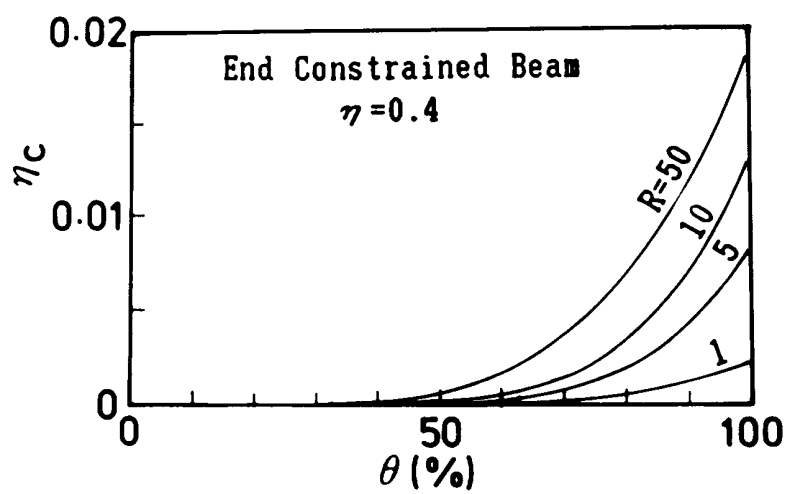

Fig. 5 Composite loss factor of the end-constrained beam $(R=10)$ the partial cover effect, the calculated results of $\eta_{c}$ and the strain energy and its density for the endconstrained beam (for the case of $R=10$ ) are shown in Figs. 5 and Fig. 6.

The value of $\eta_{c}$ does not show a peak value, and also, those of the fully covered beam decrease markedly in comparison with the center-covered beam in Fig. 3. These contrasting features of $\eta_{c}$ are clearly caused by the constraint of the viscoelastic layer ends. This constraint causes the decrease of $U_{s}$ over the entire beam range, especially in the neighbor of the covered ends (here, $U_{s}$ of the $60 \%$-covered beam is not observed to be extremely small), and causes the increase of $U_{e}$ depending on the increase of $u_{5}$.

\section{3 Separately covered beam}

It is clear by means of the previous discussion

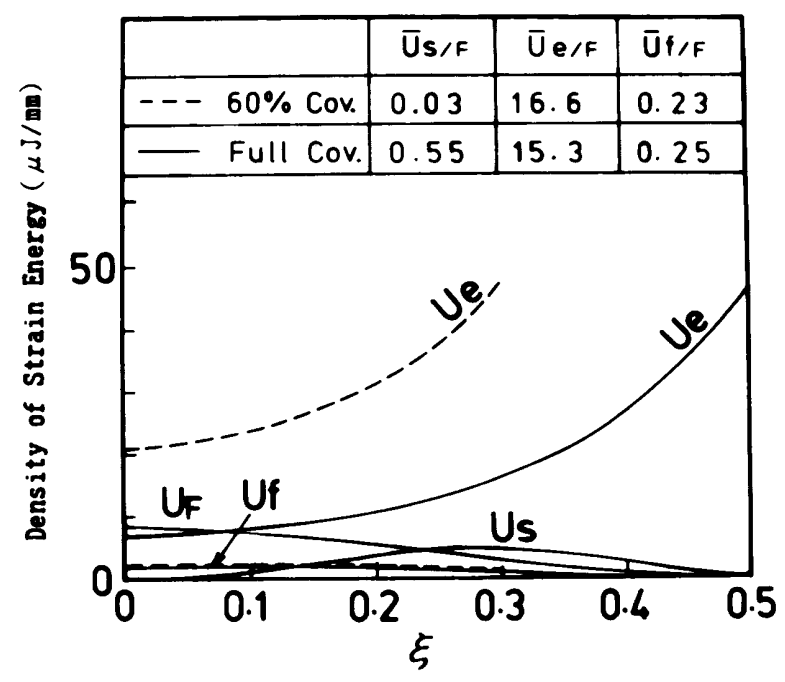

Fig. 6 Strain energy and its densities of the end-constrained beam $(R=10)$

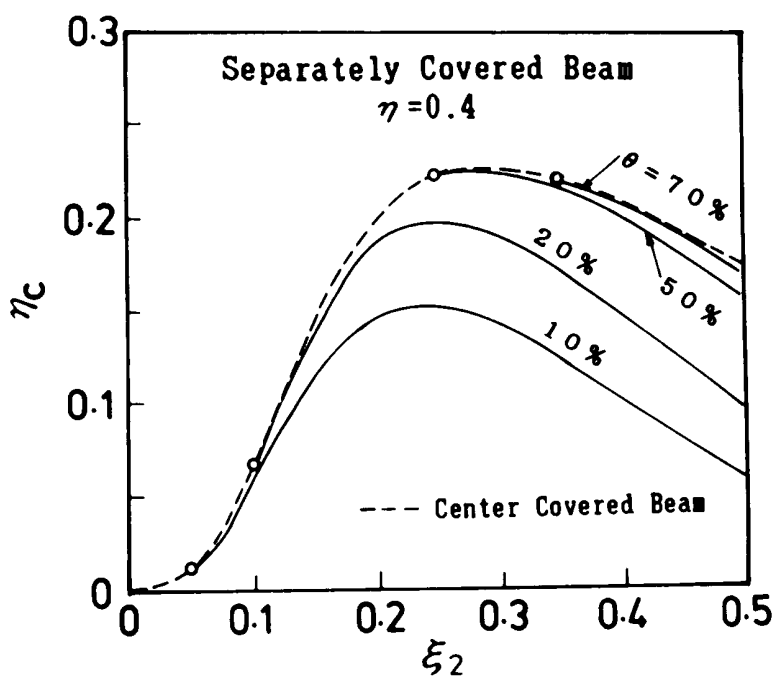

Fig. 7 Composite loss factor of the separately covered beam $(R=10)$ 
that the partial cover effect depends on the strain energy dissipation of the viscoelastic Layer ends. In order to develop such an end effect, in this paragraph, we discuss the calculated strain energy and its density for the separately covered beam in Fig. 7. The abscissa in Fig. 7 is the dimensionless location of the outer viscoelastic layer end $\xi_{2}\left(\xi_{2}=l_{2} / 2 L\right)$, the cover ratio $\theta$ $\left(\theta=2\left(\xi_{2}-\xi_{1}\right)\right.$, hare $\xi_{1}$ is $\left.\xi_{1}=l_{1} / 2 L\right)$ is a parameter, and the dotted line shows the results of the center-covered beam with the same outer viscoelastic layer end.

If the girder part becomes wider, the value of $\eta_{c}$ becomes higher than that of the center-covered beam with the same cover ratio (shown as points in the dotted line), and takes a peak value at its optimum span width when its cover ratio is under vange of the optimum cover ratio of the center-covered beam. In the case of $\theta=20 \%$, for example, $\eta_{c}=0.198$ at the optimum separation $\left(\xi_{2}=0.25\right)$, in contrast with $\eta_{c}=$ 0.07 at the center-covered beam $\left(\xi_{2}=0.1\right)$. This peak value gradually becomes closer to $\eta_{c \max }$ of the optimum center-covered beam in accordsnce with the increase of the cover ratio $\theta$. On the other hand, the increase of the cover ratio (for example $\theta=70 \%$ ) over that of the optimum center-covered beam $(\theta=60 \%)$, the value of $\eta_{c}$ does not take the peak value and decrease in accordance with the increase of the span width.

In order to discuss these damping characteristics, the strain energies in the case for $\theta=20 \%$ are shown in Fig. 8, varying the location of the outer covered layer end such as $\xi_{2}=0.1$ (the case of no separation), $0.2,0.3,0.4$, and 0.5 (the case locating the outer ends to the elastic beam ends). In Fig. 8, the values of $U_{s}$ of the outer and the inner ends become higher due to the

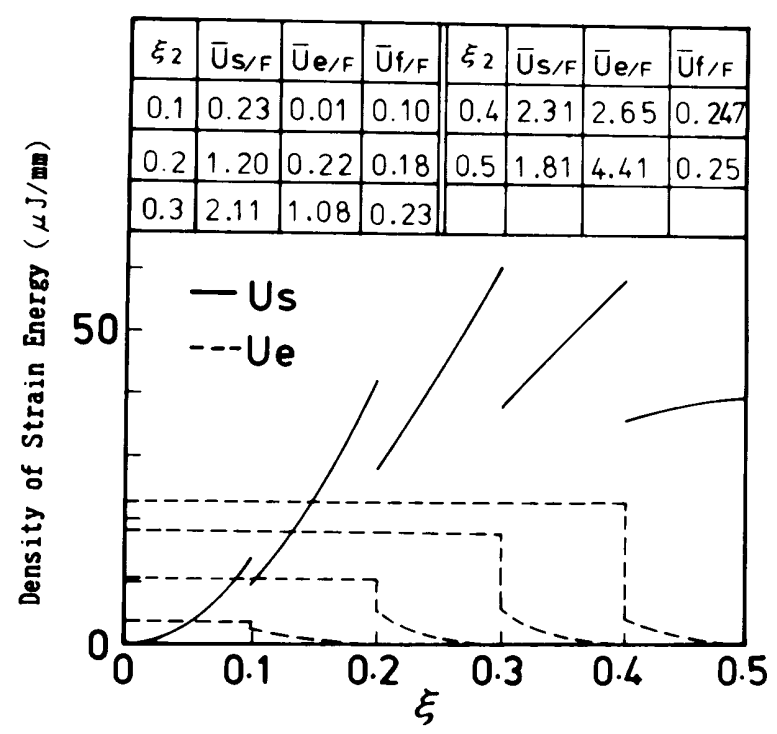

Fig. 8 Strain energy and its densities of the separately covered beam $(R=10, \theta=20 \%)$ separation (as shown in $\xi_{2}=0.2 \sim 0.5$ ) than those of the center-covered beam (as shown in $\xi_{2}=0.1$ ), and take the maximum values at $\xi_{2}=0.4$. Therefore, the separation of the viscoelastic layer results in a higher value of $\eta_{c}$ than those of without separation due to the increase of $U_{s}$ of both outer and inner ends. However, the separation over the optimal span length results in lowering the value of $\eta_{c}$ due to the decrease of $U_{s}$ and the increase of $U_{e}$ caused by longer girder part. From the above features of both $U_{s}$ and $U_{e}$, it is known that there is an optimum separation width to make $\eta_{c}$ a peak value.

The similar calculated results for the other case with the large cover ratio (the case of $\theta=70 \%$ in Fig. 7 , which is not illustrated here) indicate that the values of both $U_{s}$ and $U_{e}$ are almost equivalent to those without separation ; then, the value of $\eta_{c}$ in this case becomes slightly lower than those of the centercovered beam, as shown in Fig. 7, due to the increase of $U_{e}$ of the girder part. Therefore, the separation for the beam with a larger cover ratio is not effective.

\section{Experiment}

In order to confirm the partial cover effect, we carried out the following experiments for the three types of partially covered beam.

\section{1 Experimental procedure}

Both the elastic and the constraining layers are used the 420 stainless steel beam (SUS420) with dimensions (width $\times$ thickness) of $10 \mathrm{~mm} \times 2 \mathrm{~mm}$ and $10 \mathrm{~mm} \times 1 \mathrm{~mm}$, respectively. The viscoelastic layer material was prepared from a commercially available damping rubber (Hamadamper $C-1$ : YOKOHAMA Rubber $\mathrm{Co}_{0}$ ), and its material constants were measured by the previously reported method ${ }^{(13)}$ at $20^{\circ} \mathrm{C}$ to be $G_{v}=40 \mathrm{MPa}$ and $\eta=0.4$. These materials were bonded to the center-covered and the separately covered beams by the adhesive (Struct Bond EH-455: MITUI-TOUATU Co.) at room temperature and were cured for five hours by a hot-press device at $40^{\circ} \mathrm{C}^{(14)}$.

The end-constrained beam specimens were utilized the same center-covered beams of which the covered ends are constrained by small chips (SUS 420 : $10 \mathrm{~mm}$ in length and $2 \mathrm{~mm}$ in thickness), and reinforced by bonding thin phosphor bronze sheets $(15 \mathrm{~mm}$ in length and $0.2 \mathrm{~mm}$ in thickness) between the chip and the constraining leyer.

A coupled vibration of the elastic layer and the girder part in the separately covered beam occurred when the girder span length became wider. In this case, several small rods $1 \mathrm{~mm}$ in diameter were inserted and bonded between these layers.

The amplitude decay curves in the damped free 
vibration of the test beams, which were simply supported end condition and $300 \mathrm{~mm}$ in the span length, were measured at $22 \pm 1^{\circ} \mathrm{C}$ in an environmental chamber using an electromagnetic exciter and an eddycurrent-type gap sensor. After recording these decay curves on a digital transient memory, the composite loss factors $\eta_{c}$ of these beams were calculated from the output data of these curves. An example of these decay curves for the test beams $(\theta=20 \%)$ are shown in Fig. 9.

\subsection{Experimental results}

Nine test beams were prepared for both the center-covered and the end-constrained beams $(R=$ $17.1)$, varying the cover ratio ranging from $10 \%$ to

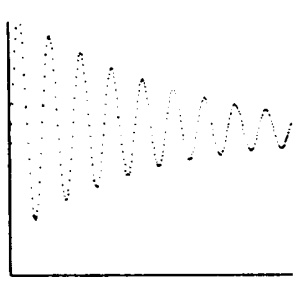

(a) the center-covered beam $\left(\eta_{c}=0.064\right)$

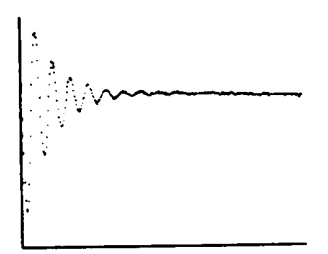

(c) the separately covered beam at the optimum $\left(\xi_{2}=0.35, \eta_{c}=0.47\right)$

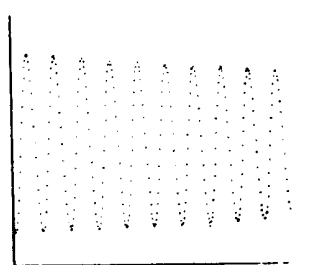

(b) the end-constrained beam $\left(\eta_{c}=0.0064\right)$

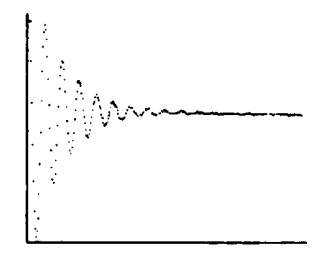

(d) the separately covered beam $\left(\xi_{2}=0.47, \eta_{c}=0.199\right)$
Fig. 9 Typical amplitude decay curves of the partially covered beams $(R=17.1, \theta=20 \%)$

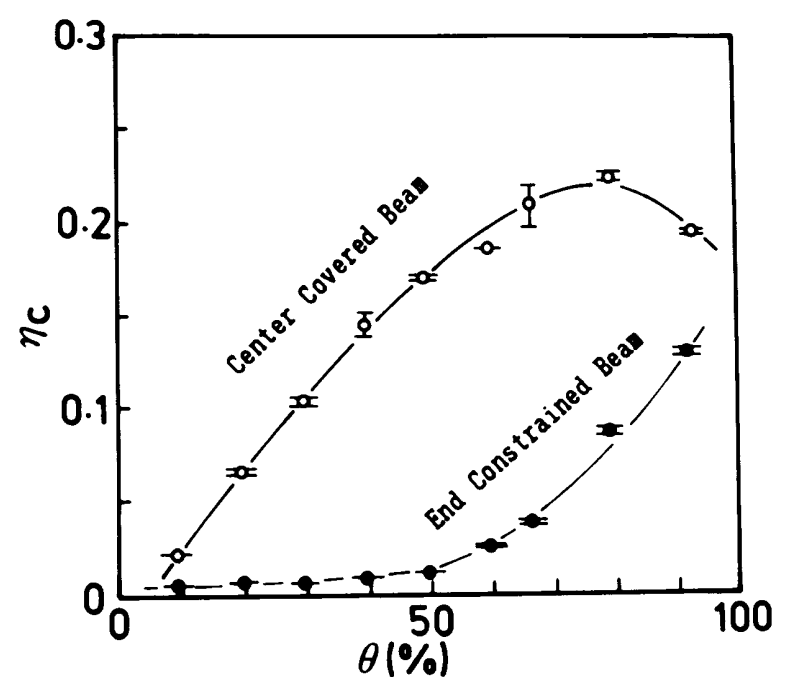

Fig. 10 Experimental results of $\eta_{c}$ for both the centercovered beam and the end-constrained beam
$90 \%$, respectively. Figure 10 shows the experimental results for both beams. The value of $\eta_{c}$ of the centercovered beam shows a pesk value, but this peak value is shifted to a higher cover ratio side in comparison with the calculated result shown in Fig. 3. The reason of this difference is attributed to the fact that the calculated result is overestimated for the shear deformation of the viscoelastic layer based on assumption ( 3 ). On the other hand, the measured value of $\eta_{c}$ of the end-constrained beam becomes over ten times greater than that of the calculated result in Fig. 5. This difference is not clear now, which may be due to the superimposition of both damping effects of an unstable constraint by the chips and of the phosphor bronze sheets. However, both calculated and measured results agree well with each other qualitatively.

The composite loss factor for the end-constrained beams with $20 \%$ and $70 \%$ cover ratio is shown in Fig. 11. The dotted line in Fig. 11 indicates the same experimental results of the center-covered beam as those in Fig. 10. These results agree well with the calculated results in Fig. 7, except for the difference of the cover ratio corresponding to the peak value of $\eta_{c}$. Therefore, the separately covered beam with a low cover ratio can obtain the highest composite loss factor by means of the optimum separation (for example, in the case of $\theta=20 \%$, the value of $\eta_{c}$ at $\xi_{2}=$ 0.35 takes $90 \%$ of $\eta_{c \max }$ of the optimal center covered beam).

From the previous discussions, the damping generation mechanism for the partial cover effect is mainly due to the shear strain energy dissipation in the neighbor of the covered viscoelastic layer ends, and also such end effect may be explained the damping mechanism of the fully covered beam in the same

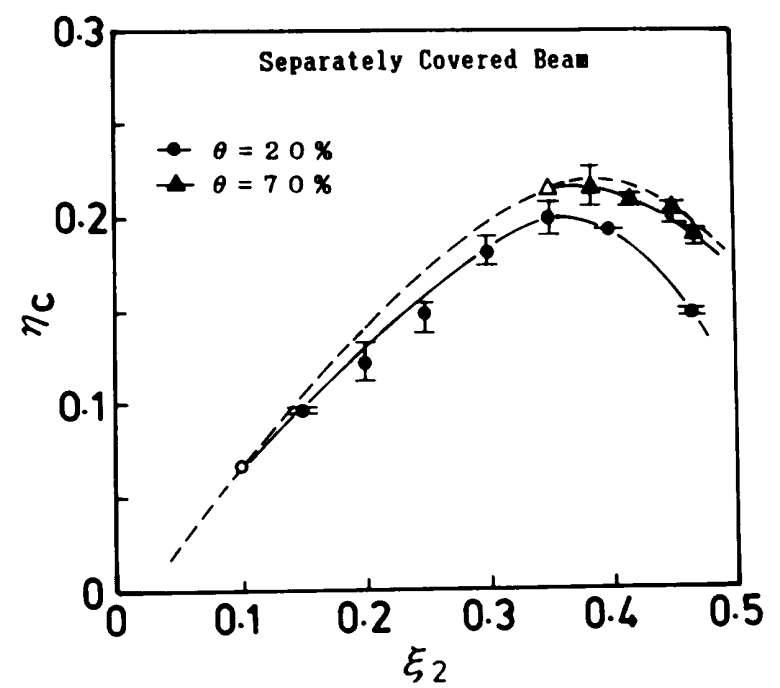

Fig. 11 Experimental results of $\eta_{c}$ for the separately covered beam 
manner.

\section{Conclusions}

An approximate strain energy analysis was carried out for three types of partially covered beam in order to explain the partial cover effect, and these damping characteristics were verified by some flexural free vibration experiments. The results obtained are summarized as follows :

(1) The partial cover effect does not occur for the end-constrained beam, and its composite loss factor becomes sufficiently lower than the centercovered beam. This fact indicates that the partial cover effect is caused by the shear strain energy dissipation in the neighbor of the covered viscoelastic layer ends.

(2) The above energy dissipation depends on both the shear and the tensile stiffnesses of the viscoelastic and the constraining layers, respectively. These facts suggest that the partial cover effect does not appear for the beam with a soft viscoelastic material, and also that such effect depends on the tensile stiffness of the constraining layer if both geometrical beam composition and viscoelastic material constants are fixed.

(3) A separation of the viscoelastic layer for a center-covered beam generates a higher composite loss factor than that without separation, and maximizes the composite loss factor by the optimal separation. But for the case of a larger cover ratio over the optimal one of the center-covered beam, the separation does not generate a higher composite loss factor than that without separation.

\section{References}

(1) Okamura, K., Automobile Technology, Soc. Automobile Tech. Jpn, (in Japanese), Vol. 41, No. 4 (1987), p. 729.

( 2 ) Oberst, H., Über Die Dămpfung Der Biegeschwingungen Dünner Bleche Durch Fest Haftende Bel ăge, Acustica 2, Akus. Beih., No.4(1952), p. 181.
(3) Kewin, E. M., Damping of Flexural Waves by a Constrained Viscoelastic Layer, J. Acoust. Soc. Am. Vol. 31, No. 7(1959), p. 952.

(4) DiTaranto,R.A.,Theory of Vibratory Bending for Elastic and Viscoelastic Layered Finite-Length Beams, Trans. ASME, J. App. Mech. Vol. 32 (1969), p.881.

(5) Mead, D. J. and Markus, S., The Forced Vibration of a Three-Layer Damped Sandwich Beam Arbitrary Boundary Conditions, J. Sound Vib., Vol.10 ( 2 ) (1969), p. 169.

(6) Markus, S., The Effect of a Stiff Dissipative Core upon the Energy Losses in a Sandwich Beam, Acta Tech., Vol. 33, No. 6 (1976), p. 655.

(7) Nokes, D. S. and Nelson, F. C., Constrained Layer Damping with Partial Coverage, Shock and Vib. Bull., Vol. 38, Pt3(1968), p. 428.

(8) Markus, S., Damping Mechanism of Beams Partially Covered by Constrained Viscoelastic Layer, Acta Tech., Vol. 31, No2(1974), p. 179.

(9) Tatemichi, A., Urata, Y. and Yasuda, H., A Vibration Analysis of the Partially Covered Multi -Layered Beam with Viscoelastic Cores, Trans. Jpn. Soc. Mech. Eng.,(in Japanese), Vol. 40, No. 335 (1974), p. 1899.

(10) Okazaki, A., Urata, Y., Ookouchi, T. and Tatemichi, A., Vibration Analysis of a Partially Covered Beam with a Constrained Viscoelastic Layers, Trans. Jpn. Soc. Mech. Eng.,(in Japanese), Vol. 53, No. 486(1987), p. 300.

(11) Lall, A. K., Asnani, N. T. and Nakra, B. C., Vibration and Damping of Rectangular Plate with Partially Covered Constrained Viscoelastic Layer, Trans. ASME, J. Vib. Acoust. Stress Reliab. Des., Vol. 109(1987), p. 241.

(12) Okazaki, A., Urata, Y., Ookouchi, T. and Tatemichi, A., A Vibration Analysis of a Partially Covered Circular Plate with a Constrained Viscoelastic layers, Trans. Jpn. Soc. Mech. Eng., (in Japanese), Vol. 53, No. 487 (1987), p. 567.

(13) Dewa, H., Determination of the Complex Shear Modulus of an Adhesive Layer by the Composite Beam Test Method, JSME Int. J., Ser. 1. Vol. 31, No. 1(1988). p. 132.

(14) Sakata, K., Adhesion Technology, Adhesion Soc. Jpn.,(in Japanese), Vol. 5, No. 2(1985), p. 11. 